\title{
AXAF IN CONTEXT: A REVOLUTION
}

\author{
MARTIN ELVIS \\ Harvard-Smithsonian Center for Astrophysics \\ 60 Garden St., Cambridge MA, 02138, USA
}

\section{AXAF and the Promise of X-ray Astrophysics}

AXAF has been a long time, 20 years, in coming. This has made AXAF so overfamiliar that it is hard to see how revolutionary it still is. Some even describe AXAF as the end of the line in X-ray technology; a never to be repeated venture to high resolution. This view is wrong. To see why we need to see where X-ray astrophysics is going.

It should not be controversial to say that X-ray Astrophysics has barely begun. Today's X-ray satellites have scored many successes; yet astronomers still extract very little of the information carried to us by X-ray photons; the subarcsecond spatial, and $R=1000$ spectral resolution routinely available at longer wavelengths, is not even begun in X-ray astronomy. AXAF is our first step into that wo ld.

Eventually X-ray telescopes will be built that have all three qualities needed to fully inhabit X-ray astrophysics: many sq. meters of effective area; sub-arcsecond angular resolution; and $R=1000-10,000$ spectral resolution. Then the riches of the atomic transitions in the X-ray band spectrum can be exploited for all clases of X-ray source (Elvis \& Fabbiano 1996). Getting there is the problem.

The next NASA Great Observatory, the Advanced X-ray Astrophysics Facility takes the first step, combining two qualities: sub-arcsecond imaging $\left(0.5^{\prime \prime}\right.$ HPD) with high spectral resolution $(R=1000)$, albeit with a modest increase in area $\left(\sim 0.1 \mathrm{~m}^{2}\right)$. So AXAF will show us where X-ray astrophysics can go. In this context we can recognize AXAF for what it is - a revolution. This short paper tries to show just how revolutionary. 


\section{The Power of AXAF}

Why is AXAF 'Advanced'? The AXAF optics put AXAF in a separate league from all other X-ray missions- past, present or planned: $75 \%$ of power within 1 arcsec diameter up to $10 \mathrm{keV}$. (This above spec. performance is thanks to Hughes-Danbury, Kodak, and the AXAF Mirror Scientist, Leon van Speybroeck). The AXAF beam area (the important quantity) is $\frac{1}{100}$ that of the ROSAT HRI, $\frac{1}{1000}$ of XMM and $\frac{1}{10,000}$ of ASCA. As a result AXAF has unprecedented sensitivity and resolution, angular and spectral.

AXAF has essentially zero background ( $\sim 1$ count/megasec/sq.arcsec) and hence high sensitivity. For point sources observations up to 2 weeks long will be photon limited. In 5 minutes AXAF will reach 10 times fainter than the ROSAT All Sky Survey, and will return a $\frac{1}{2}$ " position. At this flux there are 4 million sources available. The deepest, megasecond, AXAF surveys will reach $f_{x} \sim 5 \times 10^{-17}$ c.g.s., about $\frac{1}{20}$ of the ROSAT limit. This is uncharted territory. Our only guide is the ROSAT fluctuations analysis (Hasinger et al., 1993). This shows that we can confidently expect several thousand sources per square degree, i.e. about 1/sq.arcmin, or 250 per ACIS-I field. But what are these objects?

Whenever one can image $\frac{1}{100}$ times the detail of any previous telescope extraordinary things will be found. The step from ROSAT to AXAF is equal to that from ground-based astronomy to Hubble Space Telescope. Hubble images often give me the feeling of looking up the answer in the back of the book. These images tell us that there is structure on every scale in astrophysics. Many of the same Hubble objects are also bright X-ray sources. Surely they won't lose all structure when we look with X-rays?

To get a more terrestrial perspective on what better angular resolution can do, consider these images from spy satellites published in the New York Times (February 10 1997, page 1.) The ratio of resolution in the three images correspond (from top to bottom) to the ROSAT PSPC, the ROSAT HRI, and AXAF. While the ROSAT HRI shows a peculiar and puzzling
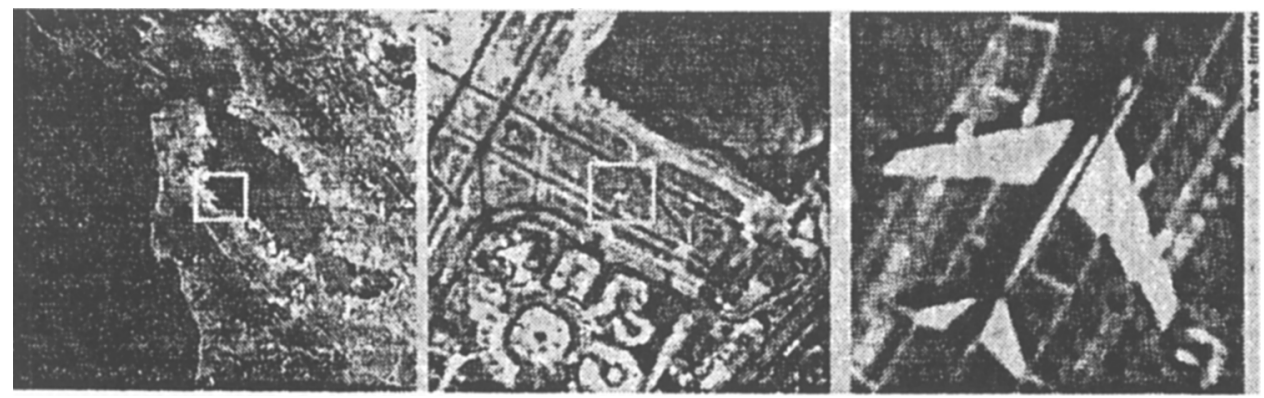

Figure 1. San Francisco Bay, SFO airport 747 at 30, 10, 1-meter resolution [N.Y.Times] 
structure inside the large feature found in the PSPC, time-resolved imaging at AXAF resolution of the cross-shaped object in the lower panel makes everything clear. Hubble shows that astrophysics works the same way.

Choosing how to image a particular object with AXAF is complex. AXAF carries three imaging instruments with multiple modes: 2 types of CCD (P.I. G. Garmire, Penn State) optimized for (1) low energy $(\mathrm{E}<0.5 \mathrm{keV})$ response and high throughput (ACIS-S); (2) good energy resolution $(\Delta E \sim 80 \mathrm{el}$ and large $\left(16^{\prime} \times 16^{\prime}\right)$ field-of-view (ACIS-I); and an HRC microchannel plate (P.I. S. Murray, SAO) which is best for fine detail $\left(<0.5^{\prime \prime}\right)$, wide field $\left(\sim 25^{\prime}\right.$ dia.), and high time resolution (msec). The ACIS (CCD) instrument gives 23-6 times, and the HRC $\sim$ twice, the PSPC or SIS count rate.

The AXAF transmission grating spectrometers give the first high resolution data that other astronomers would call 'spectra', rather than broadband photometry, with useful area. The AXAF gratings have 100 times the spectral resolution of the ASCA SIS, $(R=\mathrm{E} / \Delta E \sim 1000)$ at $1 \mathrm{keV}$, and cover a seven times broader energy range, $0.07-10 \mathrm{keV}$. The line blending problems that limit current spectra are largely gone at this resolution, opening up fainter lines and so many physical diagnostics. The low energy (LETGS, P.I. B. Brinkmann, Utrecht) and high energy (HETGS, P.I. C. Canizares, MIT) AXAF gratings have 20-200 times greater area than their predecessors on Einstein. At high energies (E>0.5 keV) the HETGS gives count rates similar to the ROSAT PSPC. The LETGS includes a region $(0.07<\mathrm{E}<0.5 \mathrm{keV})$ only previously explored by the EUVE SWS spectrometer, and has an area some 10 times larger.

AXAF is the first X-ray telescope with good simultaneous spatial and spectral resolution. Each ACIS CCD chip will have 250,000 independent beam areas. (ASCA has perhaps 16.) Not that AXAF has the effective area to fill so many bins, but in a complex source it will be possible to isolate structures, even the sinuous shock fronts in supernova remnants and clusters of galaxies, and derive their distinctive spectra.

The transmission gratings have spatial resolution too. They are slitless spectrographs, familiar from optical prism surveys. The image of the source is diffracted, so e.g. a supernova remnant makes an image in each of the lines of its spectrum. Complex fields can provide enormous returns of data. A stellar cluster can yield dozens of spectra. This is no simple analysis task - the spectra overlap in space. Fortunately the CCD energy resolution provides a third axis, and in this data cube the spectra will almost all thread delicately past one another.

With AXAF's order of magnitude advances in angular resolution, in spectral resolution and in both at once we can expect surprises. Complex spectra will show up in unusual places; many spectra will show features that are simply unknown, since laboratory work has covered only a few of the 
transitions we will encounter with AXAF; and many sources now thought of as simple will show complex images, even whole new types of source. For example, Hubble has shown that the bright stars in the Orion Trapezium are surrounded by evaporating proto-planetary disks around nearby, newly forming stars (Bally et al., 1997). Since the bright stars are also bright Xray sources, it is a simple prediction that these evaporating proto-planetary disks will be shining in fluorescent $\mathrm{X}$-rays.

\section{AXAF is a Culmination, AND a Beginning}

Both technologically and scientifically AXAF is like Hubble: both achieved 10 times improved resolution by using heavy, rigid mirrors and were limited in area by the mirror weight. But both demonstrate that high resolution is possible, not end of the line. Scientifically, both let us see how complex, yet comprehensible, the universe is. There will be no going back to less resolution, once we have seen Hubble and AXAF images.

How do we get to more area with high resolution? Which axis should we push on first? A first step is HTXS. With $\sim 3$ sq.meters of collecting area it pushes the area dimension hard, maintaining good spectral resolution. However angular resolution will be limited. What is next?

The stumbling block is $\mathrm{X}$-ray optics: we need 10 sq.meters of effective area, yet must maintain arcsecond resolution (Elvis \& Fabbiano 1996) and be light in weight. Work on this challenging goal is beginning in Europe, under the 'XEUS' banner (M. Turner, these proceedings). Discussions in the NASA community are just beginning. Certainly if we do not begin to develop the technology for such a mission X-ray astronomy will wait another 20 years before fulfilling the promise of the AXAF revolution.

\section{Your Turn}

After launch AXAF will quickly become a user driven observatory. PV and Cal. observations will be public at once. From month 5 onward $70 \%$ of the time will be for Guest investigators, increasing later to $85 \%$. About $12 \mathrm{Msec}$ of observing time is up for bids in the first NASA announcement of opportunity, with a deadline of 2 February 1998. A Proposers Guide and an AXAF simulator ('MARX') guide are available from the AXAF Science Center (http://asc.harvard.edu). AXAF is here at last. Enjoy it.

This work was supported in part by NASA contract NAS8-39073 (ASC).

Bally J., et al. 1997, http://www.cita.utoronto.ca/ johnston/orion.html

Elvis M., \& Fabbiano G., 1996, in 'Next Generation X-ray Observatories', [U. Leicester], eds. M.J.L.Turner. \& M.G.Watson XRA97/02, p. 33

Hasinger G. et al., 1993, A\& A 288, 466 